\title{
Correction to: Survey indicated that core outcome set development is increasingly including patients, being conducted internationally and using Delphi surveys
}

Alice M. Biggane ${ }^{1,2^{*}}$, Lucy Brading ${ }^{3}$, Philippe Ravaud ${ }^{4,5,6,7}$, Bridget Young ${ }^{8}$ and Paula R. Williamson ${ }^{9}$

\section{Correction}

In the original publication [1] the abbreviation for Core outcome sets (COS) was pluralized unnecessarily throughout the next. The original article was updated to rectify this error.

\begin{abstract}
Author details
'Department of Biostatistics, University of Liverpool, Liverpool, UK. ${ }^{2}$ INSERM, U1153 Epidemiology and Biostatistics Sorbonne Paris Cité Research Center (CRESS), Methods of Therapeutic Evaluation of Chronic Diseases (METHODS) Team, Paris Descartes University, Sorbonne Paris Cité, Paris, France. ${ }^{3}$ Institute of Psychology Health and Society/North West Hub for Trials Methodology Research, University of Liverpool, Liverpool, UK. ${ }^{4}$ Centre de Recherche Epidémiologie et Statistique, INSERM U1153, Paris, France. ${ }^{5}$ Cochrane France, Paris, France. ${ }^{6}$ Centre d'Épidémiologie Clinique, Hôpital Hôtel-Dieu, Assistance Publique-Hôpitaux de Paris, 75004 Paris, France. ${ }^{7}$ Department of Epidemiology, Mailman School of Public Health, Columbia University, New York, NY, USA. ${ }^{8}$ Department of Psychological Sciences and MRC North West Hub for Trials Methodology Research, University of Liverpool, Liverpool, UK. ${ }^{9}$ MRC North West Hub for Trials Methodology Research, Department of Biostatistics, University of Liverpool, Liverpool, UK.
\end{abstract}

Published online: 08 August 2018

\section{Reference}

1. Survey indicated that core outcome set development is increasingly including patients, being conducted internationally and using Delphi surveys. Trials. 2018;19:113. https://doi.org/10.1186/s13063-018-2493-y.

\footnotetext{
* Correspondence: abiggane@liverpool.ac.uk

'Department of Biostatistics, University of Liverpool, Liverpool, UK

${ }^{2}$ INSERM, U1153 Epidemiology and Biostatistics Sorbonne Paris Cité Research

Center (CRESS), Methods of Therapeutic Evaluation of Chronic Diseases

(METHODS) Team, Paris Descartes University, Sorbonne Paris Cité, Paris,

France
} 